\title{
Temporal and Geographical Trends of Incidence of Thyroid Cancer in Golestan, Iran, 2004-2013
}

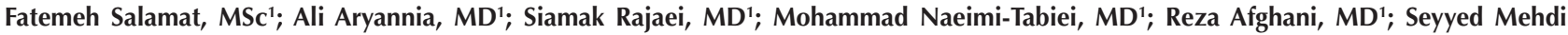 \\ Sedaghat, MD²; Esmaeil Naeimi-Tabiei, MD³; Faezeh Salamat, MSc'; Reza Hosseinpour, MSc²; Abdolreza Fazel, MD $^{4 *}$; Marzieh Araghi, PhD; \\ Gholamreza Roshandel, $\mathrm{PhD}^{6,4}$
}

${ }^{1}$ Cancer Research Center, Golestan University of Medical Sciences, Gorgan, Iran ${ }^{2}$ Deputy of Public Health, Golestan University of Medical Sciences, Gorgan, Iran ${ }^{3}$ Department of Pathology, Sayyad Shirazi Hospital, Golestan University of Medical Sciences, Gorgan, Iran ${ }^{4}$ Golestan Research Center of Gastroenterology and Hepatology, Golestan University of Medical Sciences, Gorgan, Iran ${ }^{5}$ Section of Cancer Surveillance, International Agency for Research on Cancer, 150 Cours Albert Thomas, Lyon, France ${ }^{6}$ Omid Cancer Research Center, Omid Preventive Medicine and Heath Promotion Center, Golestan University of Medical Sciences, Gorgan, Iran

\begin{abstract}
Background: Thyroid cancer is the most common type of endocrine cancer. We aimed to determine the incidence rates of thyroid cancer across a 10-year period (2004-2013) in Golestan, Iran.

Methods: We obtained the thyroid cancer data from Golestan Population-Based Cancer Registry (GPCR). Age-standardized incidence rates (ASR) were calculated and reported per 100000 person-years. The Joinpoint software was used to assess time trends, and average annual percent changes (AAPCs) and their corresponding 95\% confidence intervals (Cls) were reported. Results: Of 326 registered patients, 83 (25.5\%) were men and $243(74.5 \%)$ were women. The mean age was 51.3 and 42.6 years for males and females, respectively. Overall, the ASR of thyroid cancer was 2.2 per 100000 person-year $(\mathrm{AAPC}=2.76 ; 95 \% \mathrm{Cl}$ : -3.68 to 9.64). The test of co-incidence showed a statistically significant difference in the incidence of thyroid cancer between men (1.3) and women (3.2) $(P<0.001)$. According to our results, the ASR of thyroid cancer in western parts of Golestan is higher, including Gorgan and Aliabad cities.

Conclusion: Increasing trends in incidence rates of thyroid cancer were found in the Golestan province during the study period, especially in women. We found significantly higher rates of thyroid cancer in women. Geographical diversities were seen in incidence rates of thyroid cancer in the Golestan province. Our results may be helpful for designing further researches to investigate the epidemiological aspects of thyroid cancer in the Golestan province.

Keywords: ASR, Golestan, Incidence rate, Iran, Thyroid cancer

Cite this article as: Salamat F, Aryannia A, Rajaei S, Naeimi-Tabiei M, Afghani R, Sedaghat SM, et al. Temporal and Geographical trends of incidence of thyroid cancer in Golestan, Iran, 2004-2013. Arch Iran Med. 2021;24(1):1-6. doi: 10.34172/aim.2021.01.
\end{abstract}

Received: September 17, 2019, Accepted: October 4, 2020, ePublished: January 1, 2021

\section{Introduction}

Cancer is a major cause of death worldwide, with an estimated 9.6 million deaths in 2018. ${ }^{1}$ According to the International Agency for Research on Cancer (IARC), there are 110115 new cases of cancer in Iran, annually. ${ }^{1}$ In recent decades, the incidence and prevalence of the cancer have increased all over the world. ${ }^{2-7}$ Recent studies have shown that between 2004 and 2008, a total of 9007 new cases of cancer have been recorded according to the population-based cancer registry of northeast Iran. Agestandardized rates of 175.30 and 141.10 per 100000 person-years have been reported for females and males, respectively. ${ }^{8,9}$

Worldwide, thyroid cancer is the most common type of endocrine cancer. According to global cancer statistics, approximately 18078957 new cancer cases occur in the world every year. Of these, an estimated $4 \%$ are thyroid cancer. Thyroid cancer is the fifth cancer among women around the world, and the fourth common cancer in Iran according to global cancer statistics $2018 .{ }^{1}$ Incidence rates are higher in women than men, children than adults, and young adults than the older population. ${ }^{2}$ Previous studies have shown that thyroid cancer incidence rates are different in areas with different ethnic and geographical characteristics. ${ }^{10}$ According to Globocan 2012, the agestandardized incidence rate (ASR) of thyroid cancer was 7.3 and 3.0 in more developed areas and less developed areas, respectively. ${ }^{11}$ The ASR of thyroid cancer was 10.2 and 3.1 per 100000 person-years for females and males worldwide, respectively. ${ }^{1}$

Some important risk factors are exposure to ionizing radiation especially in childhood, age, female gender, genetic syndromes, history of goiter and history of benign nodules/adenomas, and Hashimoto's thyroiditis. Iodine 
deficiency and endemic goiter are some unproved risk factors for thyroid cancer. ${ }^{2,12}$

Access to descriptive epidemiological data on thyroid cancer may be helpful in conducting further research projects as well as in cancer controlling, especially in highrisk populations. The Golestan province of Iran is a high incidence region for upper gastrointestinal cancers. ${ }^{9,13}$ In this population-based study, we aimed to report the 10year incidence rates (2004-2013) of thyroid cancer in Golestan, Iran.

\section{Materials and Methods}

Golestan is a northern province of Iran, with a total land area of $20438 \mathrm{~km}^{2}$, which accounts for $1.3 \%$ of the total land area of Iran. The population of the province is 1868819 (2.23\% of the total population of Iran).

All primary thyroid cancers recorded in Golestan Population-Based Cancer Registry (GPCR), were enrolled in this cross-sectional study. Data collection was defined according to IARC guidelines. Details of methods of data collection and processing in GPCR were described previously. ${ }^{9,13}$

Potential sources of data included all hospitals, pathology/laboratory centers, imaging centers and selected specialist physician's offices in both public and private sectors. The information was obtained from the annually updated list of the Deputy of Treatment Affairs of the Golestan University of Medical Sciences (GOUMS).

Another source of data was the death registry unit of the health department of GOUMS. Cancer mortality data were linked to cancer incidence data to identify cases for whom the diagnosis was made by "death certificate only". To minimize underestimation due to referral of cancer cases to neighboring provinces, GPCR collects data from neighboring provinces, especially in Mazandaran, Tehran and Razavi Khorasan, on a regular basis.

Validity of the information gathering process was verified by rechecking ten percent of the questionnaires with original documents. The third edition of the International Classification of Diseases for Oncology (ICD-O) was used for coding site and morphology of the tumor ${ }^{14}$ in which code C73.9 has been assigned to thyroid cancer. Duplicate reports were checked considering different variables (patient's full name, age, father's name, tumor site, residence area and year of disease diagnosis), and were removed before entering the database. We entered the data into the CanReg software. ${ }^{15}$ We used the world standard population (18-groups) ${ }^{16}$ to calculate the ASR of thyroid cancer using the direct standardization method. ${ }^{17}$ ASR was reported per 100000 person-years. GPCR used provincial population census, which is done every year by the GOUMS health department. The list of subdivisions of the Golestan province was collected from the Statistical Center of Iran (SCI). Temporal trends in incidence rates of thyroid cancer were assessed using joinpoint regression analysis. ${ }^{18}$ We used the Joinpoint regression program version 4.6.0.0 for the joinpoint regression analysis. ${ }^{19}$ Heteroscedasticity was handled by Joinpoint using weighted least squares. Autocorrelation was taken into account by Joinpoint using the method described by Kim et al. ${ }^{18}$ Average annual percent changes (AAPCs) and their corresponding 95\% confidence intervals (CIs) were reported. The GOUMS ethics committee reviewed and approved the study protocol.

\section{Results}

During the 10-year period from 2004 to 2013, 326 (1.6\%) of the total 19807 new cancer cases reported in GPCR were thyroid cancer. The patients' characteristics including age, sex and residence area are displayed in Table 1. Eighty-three (25.5\%) cases were men and 243 (74.5\%) were women. The mean (SD) age was 51.3 (17.9) and 42.6 (16.5) years for men and women, respectively. One hundred ninety $(58.28 \%)$ patients with thyroid cancer lived in urban areas and the remaining 136 (41.72\%) patients were from rural areas. Overall, the ASR of thyroid cancer in the Golestan province was 2.25 per 100000 person-year. The ASR of thyroid cancer was more than 2.46-folds higher in women (3.2 per 100000 person-year) than men (1.3 per 100000 person-year) (Table 2). The coincidence test showed a statistically significant difference in the incidence of thyroid cancer between men and women $(P<0.001)$.

The age specific incidence rates (per 100000 personyear) of thyroid cancer in men and women during the 10year period are shown in Figure 1.

Table 2 shows the temporal trends in the ASR of thyroid cancer in men and women from 2004 to 2013. Figure 2 shows the temporal trends in the ASR of thyroid cancer in the Golestan province during the study period by gender. The AAPC were 1.49 (95\% CI: -6.23 to 9.85$)$ and 2.76 (95\% CI: -3.68 to 9.64 ) in men and women, respectively. The Joinpoint regression analysis suggested a nonsignificant increase in thyroid cancer incidence rates in women. Figure 3 shows the temporal trends in the ASR of

Table 1. Characteristics of Patients with Thyroid Cancer in Golestan Population-Based Study, 2004-2013

\begin{tabular}{lcc}
\hline Variable & Cases & $\%$ \\
\hline Age $(y)$ & & \\
$<40$ & 146 & 44.80 \\
$40-49$ & 52 & 16.00 \\
$50-59$ & 54 & 16.60 \\
$60-69$ & 47 & 14.40 \\
$\geq 70$ & 27 & 8.30 \\
Sex & & \\
Men & 83 & 25.46 \\
Women & 243 & 74.54 \\
Residence Area & & \\
Urban & 190 & 58.28 \\
Rural & 136 & 41.72 \\
\hline
\end{tabular}


Table 2. Number, Crude Rates, ASR (Per 100000 Persons-Year) and 95\% Cls for ASR of Thyroid Cancer in Urban and Rural Areas in Golestan Province of Iran during 2004-2013 by Sex

\begin{tabular}{|c|c|c|c|c|c|c|}
\hline \multirow{2}{*}{ Years } & \multicolumn{3}{|c|}{ Male } & \multicolumn{3}{|c|}{ Female } \\
\hline & Urban & Rural & Total & Urban & Rural & Total \\
\hline \multicolumn{7}{|l|}{2004} \\
\hline No. & 4 & 1 & 5 & 13 & 7 & 20 \\
\hline Crude rate & 1.1 & 0.2 & 0.6 & 3.5 & 1.7 & 2.5 \\
\hline ASR & 1.4 & 0.2 & 0.8 & 3.4 & 2.4 & 2.9 \\
\hline $95 \% \mathrm{CI}$ for ASR & $0.0-2.9$ & $0.0-0.8$ & $0.0-1.6$ & $1.3-5.5$ & $0.6-4.2$ & $1.5-4.3$ \\
\hline \multicolumn{7}{|l|}{2005} \\
\hline No. & 4 & 5 & 9 & 11 & 5 & 16 \\
\hline Crude rate & 1.0 & 1.2 & 1.1 & 2.9 & 1.2 & 2.0 \\
\hline ASR & 1.3 & 1.8 & 1.6 & 3.6 & 1.1 & 2.3 \\
\hline $95 \% \mathrm{CI}$ for ASR & $0.0-2.8$ & $0.3-3.3$ & $0.5-2.7$ & $1.2-6.0$ & $0.1-2.1$ & $1.1-3.5$ \\
\hline \multicolumn{7}{|l|}{2006} \\
\hline No. & 6 & 1 & 7 & 18 & 14 & 32 \\
\hline Crude rate & 1.5 & 1.4 & 0.9 & 4.5 & 4.8 & 3.9 \\
\hline ASR & 1.7 & 2.2 & 1 & 5.3 & 6.6 & 4.6 \\
\hline $95 \% \mathrm{CI}$ for ASR & $0.2-3.2$ & $1.9-2.5$ & $0.2-1.8$ & $2.9-7.7$ & $4.4-8.8$ & $3.0-6.2$ \\
\hline \multicolumn{7}{|l|}{2007} \\
\hline No. & 1 & 5 & 6 & 10 & 8 & 18 \\
\hline Crude rate & 0.2 & 1.2 & 0.7 & 2.5 & 1.9 & 2.2 \\
\hline ASR & 0.2 & 1.7 & 0.9 & 2.8 & 2.1 & 2.4 \\
\hline $95 \% \mathrm{CI}$ for ASR & $0.0-0.6$ & $0.0-3.5$ & $0.0-1.8$ & $1.2-4.4$ & $0.2-4.0$ & $1.2-3.6$ \\
\hline \multicolumn{7}{|l|}{2008} \\
\hline No. & 7 & 5 & 12 & 11 & 5 & 16 \\
\hline Crude rate & 1.6 & 1.2 & 1.4 & 2.6 & 1.2 & 1.9 \\
\hline ASR & 2.3 & 1.2 & 1.8 & 3.7 & 2 & 2.8 \\
\hline $95 \% \mathrm{CI}$ for $\mathrm{ASR}$ & $0.6-4.0$ & $0.1-2.3$ & $0.8-2.8$ & $1.3-6.1$ & $0.1-3.9$ & $1.3-4.3$ \\
\hline \multicolumn{7}{|l|}{2009} \\
\hline No. & 2 & 9 & 11 & 15 & 11 & 26 \\
\hline Crude rate & 0.5 & 2.1 & 1.3 & 3.6 & 2.6 & 3.1 \\
\hline ASR & 0.6 & 2.4 & 1.5 & 3.3 & 2.7 & 2.9 \\
\hline $95 \% \mathrm{CI}$ for ASR & $0.0-1.5$ & $0.6-4.2$ & $0.5-2.5$ & $1.6-5.0$ & $1.2-4.2$ & $1.7-4.1$ \\
\hline \multicolumn{7}{|l|}{2010} \\
\hline No. & 5 & 4 & 9 & 15 & 8 & 23 \\
\hline Crude rate & 1.1 & 0.9 & 1.0 & 3.5 & 1.9 & 2.7 \\
\hline ASR & 1.5 & 1.4 & 1.4 & 4 & 2.2 & 3 \\
\hline $95 \% \mathrm{CI}$ for $\mathrm{ASR}$ & $0.3-2.7$ & $0.0-3.5$ & $0.3-2.5$ & $2.0-6.0$ & $0.4-4.0$ & $1.7-4.3$ \\
\hline \multicolumn{7}{|l|}{2011} \\
\hline No. & 3 & 3 & 6 & 10 & 11 & 21 \\
\hline Crude rate & 0.7 & 0.7 & 0.7 & 2.2 & 2.6 & 2.4 \\
\hline ASR & 0.7 & 0.9 & 0.8 & 2.3 & 2.5 & 2.4 \\
\hline $95 \% \mathrm{CI}$ for ASR & $0.0-1.5$ & $0.0-1.8$ & $0.2-1.4$ & $0.9-3.7$ & $0.8-4.2$ & $1.3-3.5$ \\
\hline \multicolumn{7}{|l|}{2012} \\
\hline No. & 6 & 2 & 8 & 20 & 17 & 37 \\
\hline Crude rate & 1.2 & 0.5 & 0.9 & 4.4 & 3.9 & 4.1 \\
\hline ASR & 1.4 & 0.7 & 1.1 & 4.6 & 4.6 & 4.6 \\
\hline $95 \% \mathrm{CI}$ for ASR & $0.2-2.6$ & $0.0-1.9$ & $0.3-1.9$ & $2.5-6.7$ & $2.0-7.2$ & $3.0-6.2$ \\
\hline \multicolumn{7}{|l|}{2013} \\
\hline No. & 7 & 3 & 10 & 22 & 12 & 34 \\
\hline Crude rate & 1.5 & 0.7 & 1.1 & 4.6 & 2.7 & 3.7 \\
\hline ASR & 1.6 & 1.1 & 1.4 & 4.5 & 2.9 & 3.7 \\
\hline $95 \% \mathrm{CI}$ for $\mathrm{ASR}$ & $0.0-3.2$ & $0.0-2.4$ & $0.3-2.5$ & $2.5-6.5$ & $1.1-4.7$ & $2.3-5.1$ \\
\hline \multicolumn{7}{|l|}{ Total } \\
\hline No. & 45 & 38 & 83 & 145 & 98 & 243 \\
\hline Crude rate & 1.0 & 1.0 & 1.0 & 3.4 & 2.5 & 2.9 \\
\hline ASR & 1.3 & 1.2 & 1.3 & 3.7 & 2.7 & 3.2 \\
\hline $95 \% \mathrm{CI}$ for ASR & $0.9-1.7$ & $0.8-1.6$ & $1.0-1.6$ & $3.2-4.2$ & $2.3-3.1$ & $2.8-3.6$ \\
\hline
\end{tabular}

Note. $\mathrm{Cl}$, confidence interval; ASR, age-standardized incidence rates. 


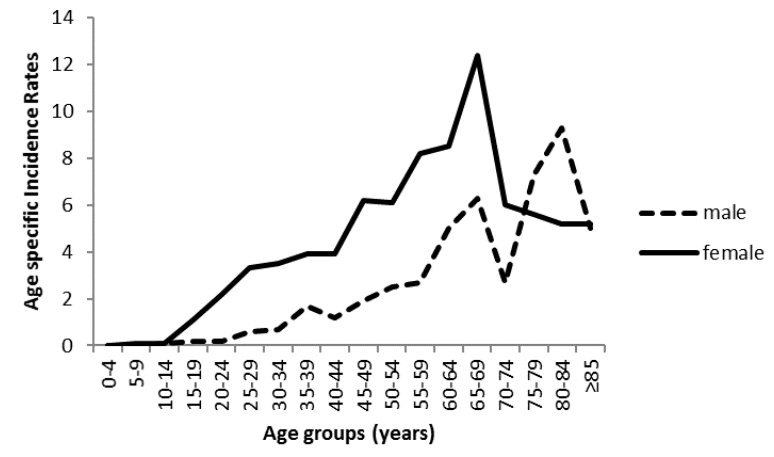

Figure 1. Age-Specific Incidence Rates (Per 100000 Persons-Year) of Thyroid Cancer in Golestan Province of Iran during 2004-2013 by Sex.

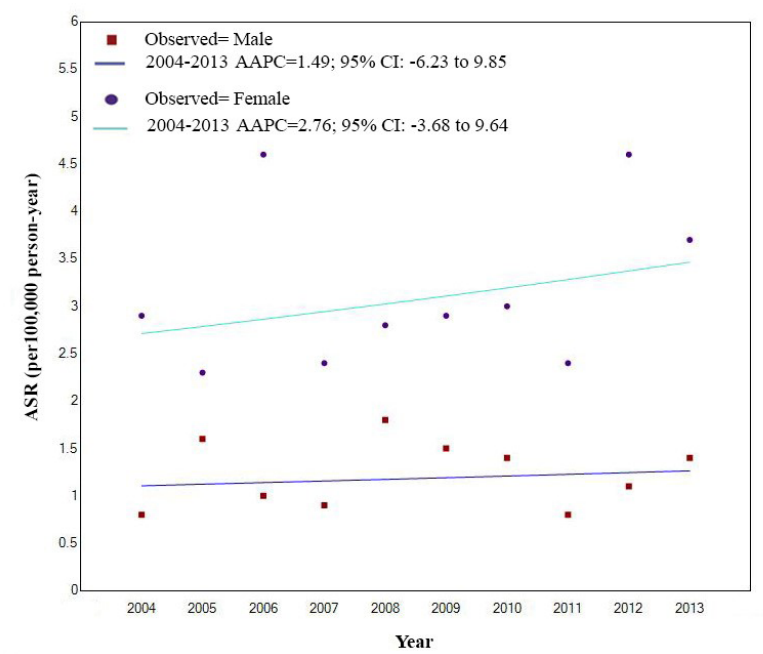

Figure 2. Trends of Age-Standardized Incidence Rates (ASR) of Thyroid Cancer in Golestan Province of Iran during 2004-2013 by Sex. AAPC, Average annual percent change; $\mathrm{Cl}$, confidence interval.

thyroid cancer in the Golestan province during the study period by residence area. The AAPC were - 0.17 (95\% CI: -6.80 to 6.90$)$ and 2.23 (95\% CI: -6.90 to 12.30$)$ in urban and rural areas, respectively. The results of joinpoint regression did not suggest a significant trend in the ASR of thyroid cancer in the two areas. The co-incidence test suggested higher incidence rates of thyroid cancer in the urban population, but the difference was not statistically significant $(P=0.25)$. Figure 4 shows the geographical distribution of thyroid cancer in Golestan cities. Our findings suggest high rates of thyroid cancer in central and western parts of the province.

\section{Discussion}

Our findings showed that $326(1.6 \%)$ of the total 19807 new cancer cases registered in GPCR from 2004 to 2013 were thyroid cancer. We found the overall ASR of thyroid cancer at 2.25 per 100000 person-year. According to global cancer statistics 2018, the incidence rates of thyroid cancer were reported at 4.3 and 6.7 in Iran and the

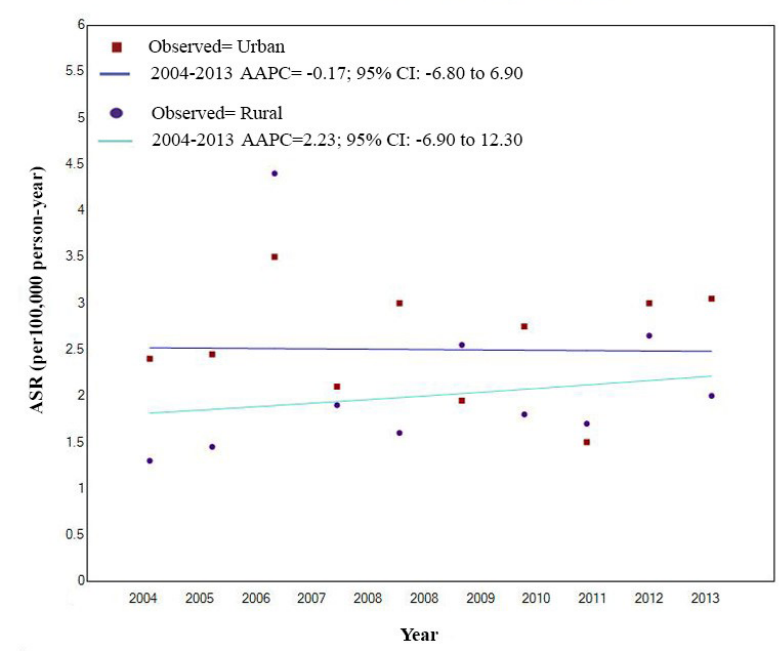

Figure 3. Trends of Age-Standardized Incidence Rates (ASR) of Thyroid Cancer in Golestan Province of Iran during 2004-2013 by Residential Place. AAPC, Average annual percent change; $\mathrm{Cl}$, confidence interval.
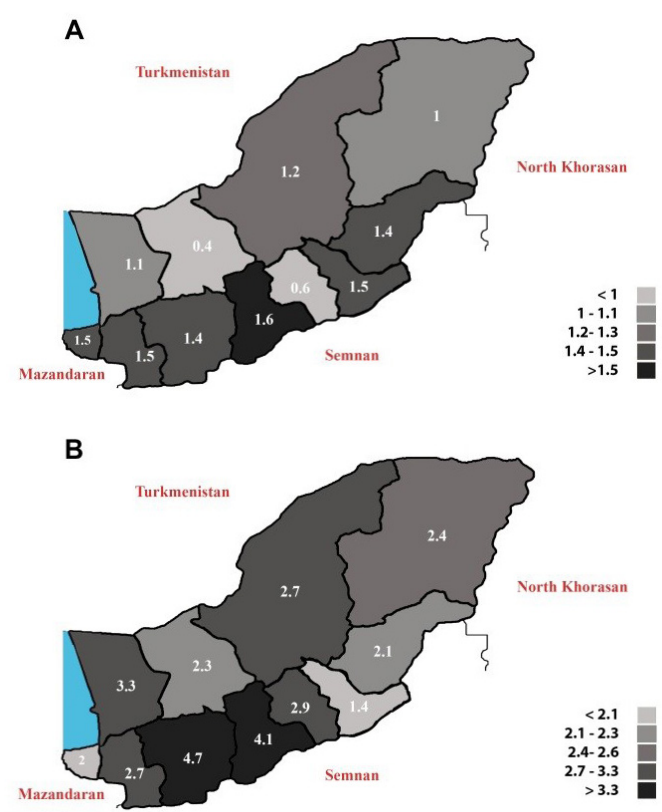

Figure 4. Age-Standardized Incidence Rates (ASR) (Per 100000 PersonsYear) of Thyroid Cancer in Subdivisions of Golestan, Iran; (A) Men, (B) Women.

globe, respectively. Generally, in global cancer statistics, the highest ASRs of thyroid cancer were found in North America $($ men $=6.9$; women $=23.1)$ and the lowest were found in West Africa $(\mathrm{men}=0.5$; women $=1.5) .{ }^{1}$ High rates of thyroid cancer were also reported for neighboring countries of Iran including Turkey ( $m e n=5.7$; women $=22.4$ ). Therefore, our findings suggest a relatively low incidence rate for thyroid cancer in Golestan, Iran. ${ }^{1}$

Our results also showed increasing trends in the ASR of thyroid cancer in Golestan during the study period, especially in women, although the changes were not 
statistically significant. The increasing trends in thyroid cancer in the Golestan province were more considerable when compared to the results of previous reports from this region. ${ }^{6,20}$ Previous reports from Iran also suggested similar increasing trends in Iran. ${ }^{21}$ A previous study in Italy showed that the thyroid cancer trend has been increasing over the years, and they concluded that according to the world thyroid cancer statistics, this trend seems to be global and not related to a specific region. ${ }^{5}$ The results of a study from China also suggested an increasing trend in the incidence of thyroid cancer between 2000 and 2012 and they concluded that it might be due to improvement in diagnosis with advanced technologies. ${ }^{22}$

As suggested by other studies, the increasing trends in the incidence of thyroid cancer in the Golestan province may be partly explained by improvements in medical care access, advanced diagnostic techniques (e.g. ultrasound examination, computed tomography and magnetic resonance imaging scanning). ${ }^{21-23}$ However, considering the rates of thyroid cancer in previous reports from this region, there may be some other explanations for this increasing trend such as exposure to environmental risk factors (e.g. medical radiation). ${ }^{2,24}$ Therefore, despite low incidence rates of thyroid cancer in our region, its trends should be closely monitored and this point should be considered in cancer programs in our population.

Our findings suggested a higher incidence rate of thyroid cancer in women compared to men. The results of the Globocan 2018 project also suggested a similar disparity in the incidence of thyroid cancer between men and women in Iran (men $=2.0$; women $=6.7$ ) as well as in the world $($ men $=3.1$; women $=10.2) .{ }^{1}$ Previous studies suggested that biological characteristics in women (e.g. pregnancy) may cause the higher incidence rate of thyroid cancer. Although the mechanism has not been clearly identified yet, it has been proposed that high rates of thyroid cancer in women may be partly related to the function of hormone receptors including androgen, estrogen, and progesterone receptors. ${ }^{25-27}$

Our finding showed the highest age-specific incidence rates for the age group 50-69 years in both males and females. Previous studies conducted in Golestan have shown that the highest incidence of thyroid cancer was in the 70-79 age groups for women and men. ${ }^{6}$ Age is a known risk factor and should be considered in controlling programs for all cancers including thyroid cancer.

This study finding showed that the highest thyroid cancer incidence rate was in the central and western parts of the Golestan province, including Gorgan and Aliabad cities. Gorgan is the capital of the province with a higher rate of urbanization and better access to healthcare services. Access to diagnostic medical services may partly explain the higher rates of thyroid cancer in this region. In addition, problems in reporting residential place may a possible explanation for this geographical diversity.
Patients who refer to Gorgan city (as a referral city) from neighboring areas may mention a temporary address instead of their permanent address in their hometown. Although this point may not be ignored, this could not have had serious effects on our results for the following reasons. First, as we mentioned in the methods section, the validity of data is routinely re-checked in $10 \%$ of the registered records for any possible errors in the process of data collection, abstraction, and data entry. Second, previous reports from this region have suggested relatively low rates of specific type of cancers including esophageal and gastric cancers in Gorgan city. ${ }^{9}$ Third, Gonbad city located in eastern part of the province is also a referral city with good access to healthcare services, but the incidence of thyroid cancer in this city is relatively lower than its neighboring cities. Finally, the thyroid cancer incidence is relatively high in Aliabad city, which is not a referral city and the situation of access to healthcare services is relatively poor compared to Gorgan and Gonbad cities. Therefore, issues in reporting residential place could not be considered a serious problem in Golestan cancer registry data and could not mainly explain the geographical discrepancies in the incidence rates of thyroid cancer in Golestan. Exposure to environmental risk factor (e.g. radiation) may also be considered as a possible explanation for the higher incidence of thyroid cancer in this part of the Golestan province. ${ }^{6}$ Further studies are recommended to assess the risk factors related to thyroid cancer in the Golestan province, especially in areas with high incidence rates including Gorgan and Aliabad cities. Geographical diversities should be considered in designing controlling programs for thyroid cancer in the Golestan province.

We found higher incidence rates of thyroid cancer in the urban population, although the difference was not significant. This finding may be explained by better access to medical services or higher exposure to risk factors of thyroid cancers in residents of urban areas. ${ }^{2,24}$ Further studies are recommended to clarify this point in our population.

In conclusion, our findings suggested an increase in the incidence rate of thyroid cancer in the Golestan province during the study period, especially in women and the rural population. Our results showed higher rates of thyroid cancer in women. We also found geographical diversities in the incidence rates of thyroid cancer in the Golestan province, with higher rates in central and western parts. Our results may be helpful for designing further researches to investigate the epidemiological aspects (e.g. risk factors, stage of disease, survival, etc.) of thyroid cancer in our population.

\section{Authors' Contribution}

FS and FS: collaborated in data analysis, wrote the first draft of manuscript; SS, SR, MN, EN and RH: collaborated in collection of data and quality control, edited and critically reviewed manuscript; $\mathrm{MA}$ and $\mathrm{AA}$ : collaborated in data processing, interpreted the results; 
edited and critically reviewed manuscript; AF conceptualized and designed the study, edited and critically reviewed manuscript; GR: conceptualized and designed the study, performed statistical analysis, edited and critically reviewed manuscript; All authors reviewed and approved the final draft of manuscript.

\section{Conflict of Interest Disclosures}

The authors declare no conflict of interest.

\section{Ethical Statement}

The study protocol was approved by the ethics committee of Golestan University of Medical Sciences.

\section{Acknowledgements}

We would like to thank our colleagues in the GPCR secretariat as well as all healthcare personals of the GPCR sources throughout the Golestan province.

\section{References}

1. Bray F, Ferlay J, Soerjomataram I, Siegel RL, Torre LA, Jemal A. Global cancer statistics 2018: GLOBOCAN estimates of incidence and mortality worldwide for 36 cancers in 185 countries. CA Cancer J Clin. 2018;68(6):394-424. doi: 10.3322/caac. 21492.

2. Khodamoradi F, Ghoncheh M, Mehri A, Hassanipour S, Salehiniya $\mathrm{H}$. Incidence, Mortality, and risk factors of thyroid cancer in the world: a review. World J Cancer Res. 2018;5(2): e1093.

3. Mathew IE, Mathew A. Rising Thyroid cancer incidence in Southern India: an epidemic of overdiagnosis? J Endocr Soc. 2017;1(5):480-7. doi: 10.1210/js.2017-00097.

4. Kitahara CM, Sosa JA. The changing incidence of thyroid cancer. Nat Rev Endocrinol. 2016;12(11):646. doi: 10.1038/ nrendo.2016.110.

5. Cossu A, Budroni M, Paliogiannis P, Palmieri G, Scognamillo F, Cesaraccio R, et al. Epidemiology of thyroid cancer in an area of epidemic thyroid goiter. J Cancer Epidemiol. 2013;2013: 584768. doi: $10.1155 / 2013 / 584768$

6. Marjani A, Kabir MJ. Incidence of thyroid cancer in Golestan province of Iran: Some initial observations. Pak J Med Sci.2008;24(6):887-90.

7. Safavi A, Azizi F, Jafari R, Chaibakhsh S, Safavi AA. Thyroid Cancer Epidemiology in Iran: a Time Trend Study. Asian Pac J Cancer Prev. 2016;17(1):407-12. doi: 10.7314/ APJCP.2016.17.1.407.

8. Taziki M-H, Fazel A, Salamat F, Sedaghat S-M, Ashaari M, Poustchi $\mathrm{H}$, et al. Epidemiology of head and neck cancers in Northern Iran: a 10-year trend study from Golestan province. Arch Iran Med. 2018;21(9):406-411.

9. Roshandel G, Sadjadi A, Aarabi M, Keshtkar A, Sedaghat SM, Nouraie SM, et al. Cancer incidence in Golestan province: report of an ongoing population-based cancer registry in Iran between 2004 and 2008. Arch Iran Med. 2012;15(4):196-200.

10. Larijani B, Aghakhani S, Haghpanah V, Mosavi-Jarrahi A, Bastanhagh M. Review of thyroid cancer in Iran. Austral-Asian J Cancer. 2005;4(4):199-203.

11. Ferlay J, Soerjomataram I, Dikshit R, Eser S, Mathers C, Rebelo $\mathrm{M}$, et al. Cancer incidence and mortality worldwide: sources, methods and major patterns in GLOBOCAN 2012. Int J
Cancer. 2015;136(5):E359-86. doi: 10.1002/ijc.29210.

12. Figge JJ. Epidemiology of thyroid cancer. In: Wartofsky L, Nostrand DV, eds. Thyroid Cancer. New York: Springer; 2016: 9-15.

13. Roshandel G, Semnani S, Fazel A, Honarvar M, Taziki M, Sedaghat $\mathrm{S}$, et al. Building cancer registries in a lower resource setting: The 10-year experience of Golestan, Northern Iran. Cancer Epidemiol. 2018;52:128-33. doi: 10.1016/j. canep.2017.12.014

14. Fritz A, Percy C, Jack A, Shanmugarathan S, Sobin L, Parkin $\mathrm{D}$, et al. International classification of diseases for oncology: ICD-O: World Health Organization; 2000. Available from: https://apps.who.int/iris/handle/10665/42344.

15. Ervik MJ, Cooke AP, Ferlay J, Rahimi A, Antomi S, Dhivar D, et al. CanReg5: Computer Software for Cancer Registries. Lyon: International Agency for Research on Cancer; 2008.

16. Segi M. Cancer Mortality for Selected Sites in 24 Countries. Nagoya, Japan: Dept. of Public Health, Tohoku University School of Medicine; 1960:1950-57.

17. Jensen OM, Parkin DM, MacLennan R, Muir CS, Skeet RG. Cancer Registration: Principles and Methods. IARC Scientific Publication No. 95. Lyon: International Agency for Research on Cancer; 1991.

18. Kim HJ, Fay MP, Feuer EJ, Midthune DN. Permutation tests for joinpoint regression with applications to cancer rates. Stat Med. 2000;19(3):335-51. doi:10.1002/(SICl)10970258(20000215)19:3<335::AID-SIM336>3.0.CO;2-Z.

19. Statistical Research and Applications Branch. Joinpoint Regression Program. Version 4.6.0.0 ed. Calverton: National Cancer Institute; 2018.

20. Parkin D, Whelan S, Ferlay J, Teppo L, Thomas D. Cancer incidence in five continents. Lyon, France: IARC Press; 2002;8:705-71.

21. Farzadfar F, Peykari N, Larijani B, Rahimzadeh S, Rezaei-Darzi E, Saeedi Moghaddam S. A comprehensive study on national and sub national trend in thyroid cancer prevalence in the iranian population, 1990-2010. J Diabetes Metab Disord. 2016;15(2):91-100.

22. Du L, Wang Y, Sun X, Li H, Geng X, Ge M, et al. Thyroid cancer: trends in incidence, mortality and clinical-pathological patterns in Zhejiang province, Southeast China. BMC Cancer. 2018;18(1):291. doi: 10.1186/s12885-018-4081-7

23. Shah JP. Thyroid carcinoma: epidemiology, histology, and diagnosis. Clin Adv Hematol Oncol. 2015;13(4 Suppl 4) :3-6.

24. Raposo L, Morais S, Oliveira MJ, Marques AP, José Bento M, Lunet N. Trends in thyroid cancer incidence and mortality in Portugal. European Journal of Cancer Prevention. 2017;26(2):135-43.

25. Kravdal ø, Glattre E, Haldorsen T. Positive correlation between parity and incidence of thyroid cancer: new evidence based on complete Norwegian birth cohorts. Int J Cancer. 1991;49(6):831-6. doi: 10.1002/ijc.2910490606.

26. Glattre E, Kravdal O. Male and female parity and risk of thyroid cancer. Int J Cancer. 1994;58(4):616-7. doi: 10.1002/ ijc. 2910580427.

27. Ron E, Kleinerman RA, Boice JD Jr, LiVolsi VA, Flannery JT, Fraumeni JF Jr. A population-based case-control study of thyroid cancer. J Natl Cancer Inst. 1987;79(1):1-12. doi: 10.1093/jnci/79.1.1. 